\title{
PRECAUTION OF HEART FAILURE BASED ON COMPUTER AIDED DIAGNOSIS SYSTEM
}

\author{
Somsubhra Gupta ${ }^{1}$ and Debasmita Mula ${ }^{2}$
}

\begin{abstract}
The objective of the work is to formulate and construct an equivalent intelligent model for the response of cardiovascular system using MATLAB 8.5 / SIMULINK application. The model is mainly made up of systemic circulation and the controller, a functional description of mechanisms working on systemic resistance, heart rate, and heart pressure and blood flow rate. The objective of this dissertation is meant to assist and enhance non medical person in their analytical studies of cardiovascular system characteristics such as distribution of blood flow, cardiac fitness, high and low blood pressure. The presented work formulates and constructs an equivalent intelligent model of the response of cardiovascular system. The model is mainly made up of best first algorithm and the bread board, a functional description of mechanisms working on systemic resistance, heart rate, heart pressure and blood flow rate. The objective of this dissertation is meant to assist and enhance non medical person in their analytical studies of cardiovascular system characteristics such as distribution of blood flow, cardiac fitness, high and low blood pressure.

Keywords - Heart precaution Algorithm, Precaution of heart failure, Preliminary Life-Guard of Heart.
\end{abstract}

\section{INTRODUCTION}

The basic question is to why medicine is prescribed by the device in spite of huge nos. of existing doctor? The idea is driven by the fact that human mind plays a key role behind maximum number of diseases. A change mind-state reflects directly on physical /mental diseases and the state of mind can change at any point of time during day / night. Here, the blood pressure area related to the heart from the whole physical system is only taken into consideration. The imbalance of pressure of blood directly affect on heart. Heart is the core of total physical system. So, if pillar of any structure is not balanced then total structure may be collapsed. The availability of the Doctors in close vicinity of the patient can't always be guaranteed. Sometimes, certain heart failure drives a patient at the door of death. Medicines prescribed by the device can regulate and prevent the worst condition till the availability of doctors. The heart, controls mind state directly by making adjustments.

In brief, heart is the pillar of physical system that influences all levels of human existence.

In the model formulation of the problem, an Artificial Intelligence based Computer aided diagnosis system designed to assist the clinical decision of non-specialist person /patient him / her-self in the analysis of probable heart failure. The system compute patient pathological condition and highlights possible symptoms of blood pressure abnormality The system based on two functional parts viz. diagnosis and follow up.

In the solution search process, an Artificial Intelligence based technique has been introduced. The diagnosis is used to detect any worse condition of blood pressure of physical status.

As a precaution, the follow-up technique is used to suggest medicine in emergent and alarming situation that is clinically approved.

Any type of heart-beat abnormality causes the heart to beat very first or very slow or unexpectedly. In that situation after the signals are filtered and parameters are extracted, the comparison are made through proposed intelligent algorithm through parametric comparison. Here the comparison is carried out by a method inspired by artificial intelligence. In this work, the artificial intelligence algorithm is implemented in java/MATLab using Simulink and

${ }^{1}$ Department of Information Technology JIS College of Engineering, Kalyani, W.B., India

${ }^{2}$ Department of Information Technology JIS College of Engineering, Kalyani, W.B., India 
the simulation results proved that the developed algorithm can be identified as a powerful technique in pre-detection of heart diseases.

In the completion phase, the developed artificial intelligence algorithm is implemented in arduino uno using $16 \mathrm{X} 2$ LCD display and the display results prove worth of identification of technique.

\section{A. Historical background of Cardio- vacular System}

Cardiac physiology or heart function is the study of healthy, unimpaired function of the heart: involving blood flow; myocardium structure; the electrical conduction system of the heart; the cardiac cycle and cardiac output and how these interact and depend on one another.

Every cell relies on surrounding interstitial fluid for oxygen, nutrients and waste disposal. And this fluid composition is kept stable through continues exchange between blood and peripheral tissue. Blood can maintain homeostasis only if stays in motion Stationary blood will exhaust of nutrients and saturated with waste product so all depends on heart Located near anterior chest wall, directly posterior to sternum Surrounded by pericardial cavity in anterior portion of mediastinum Heart beats about 100 thousand times each day, and its size is roughly the size of clinched fist Blood flows through a network of blood vessels between heart and peripheral tissues.

Those blood vessels can be subdivided into:

1. Pulmonary circuit: Take blood to lung and back to heart;

2. Systemic circuit: Take blood to peripheral tissue and back to heart.

Each circuit begins and ends in heart, and blood travels through these circuits

in sequence Arteries carry blood away from heart. Veins return blood to the heart Capillaries small thin walled vessels between the smallest arteries and veins (allow exchange) Contains 4 muscular chamber, 2 associated with each circuit The RA receives blood from systemic circuit and passes it to RV The RV pumps blood into pulmonary circuit LA receives blood from Pulmonary circuit and pass it to LVLV eject blood to systemic circuit.

\section{B. Function of the Heart}

- Spontaneously generates electrical signal (action potential).

- Conducted through cellular pathways into atrium, causing contraction of atria then ventricles.

- Repeats, maintaining 60- 100 beats per minute.

- A real-time system, and natural pacemaker

C. Abnormalities in electrical conditions

- $\quad$ Missed / slow heart beat.

- Can be corrected by implantable pacemakers.

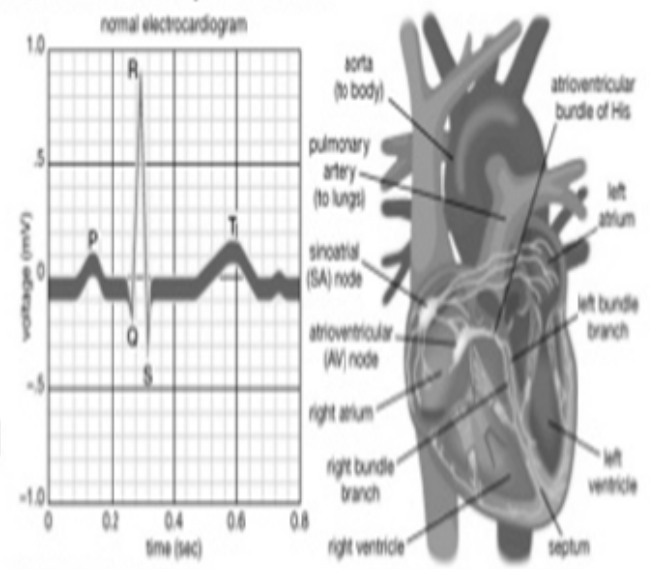

Fig. 1: Normal Electrocardiogram and human heart.

\section{Type of Cardio-Vascular Diseases}

Atherosclerosis:

Characterized by deposits of Fatty substances, cholesterol, cellular waste products, calcium, and fibrin in the inner lining of the artery.

Hyperlipidemia - abnormally high blood lipid level

Plaque - the buildup of deposits in the arteries

Coronary heart disease (CHD): 
Myocardial infarction (MI) or heart attack - blood supplying the heart is disrupted

Coronary thrombosis - blood clot in the artery

Embolus - when the blood clot is dislodged and moves through the circulatory system

Collateral circulation - if blockage to the heart is minor, an alternative blood flow is selected

Chest pain (angina pectoris):

Ischemia - reduction of the heart's blood and oxygen supply. The more serious the oxygen deprivation the more severe the pain

Nitroglycerin - drug used to relax (dilate) the veins

Irregular heartbeat (arrhythmia):

An irregularity in heart rhythm

Tachycardia - racing heart in the absence of exercise or anxiety

Bradycardia - abnormally slow heartbeat

Fibrillation - heart beat is sporadic, quivering pattern

Congestive heart failure (CHF):

Damaged or overworked heart muscle is unable to keep blood circulating normally.

Damage to heart muscle may result from rheumatic fever, pneumonia, heart attack, or other cardiovascular problem.

Lack of proper circulation may allow blood to accumulate in the vessels of the legs, ankles, or lungs.

Congenital and rheumatic heart disease:

Congenital heart disease affects 1 out of 125 children born.

May be due to hereditary factors, maternal diseases, or chemical intake (alcohol) during fetal development

Rheumatic heart disease results from rheumatic fever which affects connective tissue.

Stroke:

Occurs when the blood supply to the brain is interrupted

Thrombus - blood clot

Embolus - free flowing clot

Aneurysm - bulging or burst blood vessel

Transient ischemic attack (TIA) - brief interruptions that cause temporary impairment.

\section{Methodological Aspects AND Algorithm}

A. Core factors[Preliminary Life-Guard of Heart]

Since the methodology is more prior than the background of Therapeutic Medicinary, so the fundamental principles are directly referred and presented the case for Therapeutic Medicinary almost at the inception of modern medicine.

This established eight arguments that remain central to the theory and practice of Therapeutic Medicinary today.

These are:

1. Success in medicine (from a standalone standpoint) does not depend on attaining a predetermined level of proficiency;

2. Medicine can change and evoke physical system;

3. Low and high dose medicine can have differing effects on individuals;

4. Medicine can influence physiological processes;

5. Medicine can be harmful in treating some conditions;

6. Medicine has a wide variety of therapeutic applications, and;

7. Medicine could be useful in preventative health care.

Now the question is how does the medicine work? From the human physiological viewpoint it is believed that heart controls or sometimes says that balance the flow of blood.

Physical and mental illness means change in the state of equilibrium of physical system. Medicine is nothing but external composition that can be effectively applied to bring back original state of physical system. The right kind of medicine, helps one relax and refresh and even during course of working, medically approved medicine improves efficiency.

B. Methodological aspects

The technique is comprised of the basic qualifications of a thinking machine namely natural language understanding, knowledge representation, reasoning and learning ability. The technique will start working using a basic knowledge base to apply some deterministic and heuristic methodologies of detection with the help of inputs. Explicitly there will be four correlated inputs. These are systolic, diastolic, pulse, age.

The first will contain a map between symptom(s) to disease(s). Next one is a map between disease(s) to medicine domain(s). In both the cases the mapping will be many to many. This implies that a particular symptom may correspond to number of number of blood pressure and vice versa. This initial status of Knowledge base inputs will 
be the standard of the Production System at the time of first trial. However with increasing number of trials, i.e. more patient it treats, the knowledge base inputs will be enriched to add up huge number of new symptoms and abnormal pressure through learning only to enhance the standard of its Production system as a whole.

The entire technique is clustered into steps as described below.
1. Diagnosis
2. Follow up

Diagnosis

Step 1: The machine will either interact directly with the patient (in the case of using blood pressure sensor) or counsel via knowledge base inputs of systolic, diastolic, pulse, age of member patient party to get the list of symptoms of the disease(s) i.e which phase blood pressure that the patient is suffering from.

Step 2: From the existing knowledge base inputs of symptom to disease it will analyze (through reasoning) what can be the possible blood pressure. In this point the technique will use a checkpoint to examine how relevant is the patients feedback through backward reasoning. If some symptoms are beyond its knowledge base then it will prescribe a basic medicine "Diuretics (water pills)" to remain constant the equilibrium of heart system and also alert the patient "The problem or disease is indeterminate, Medicine cannot be determined? recognized,!!!! contact physically to nearest doctor as soon as possible."

It will be practically implemented by keeping some weights to each symptom to determine precedence as number of diseases can have same symptoms with different intensity.

\section{Follow up}

Step 3: Once the disease(s) is determined, the second follow up of disease to medicine domain will come into effect to conclude about the kind of treatment the patient should have. The medicine domain will contains several medicines according to the patient age, pulse, systolic \& diastolic sample.

Step 4: The interaction between machine and patient will occur in two stages of which the first is already discussed at step 1. This step will host the second phase of interaction through which the patients suitable medicine(i.e low or high dose according to the age, pulse, systolic \& diastolic pressure) will be analyzed. This final step will be concluded through heuristic determination of sample medine that already exists in the medicine domain.

\section{Heart precaution Algorithm}

The following comprehensive algorithm can represent the diagnosis done in the various phases and determination of medicines that are to be prescribed.

If (the concluded list of symptoms of blood pressure abnormality of interaction are all within knowledge base)

Explore symptom to blood pressure abnormality database to diagnose the disease or list of diseases. Assign specific value to the symptom and apply some heuristic function to conclude about the probable list of diseases.

If (the list of diseases are all in knowledge base i.e. no indeterminate disease)

L1: Explore disease to medicine domain database to determine the specific Medicine domain of treatment. Assign static evaluation function for medicine domain to compute most-fit if more than one medicine is significant.

Enable phase 2 of interaction to conclude about the adoptation of medicine component at this certain age of the patient.

If (patients adaptable medicine is there in the knowledge base)

Use that information to prescribe specific medicine for treatment.

Else

Analyze most close-fit for treatment using some heuristic Function. If it be indeterminate then prolonged phase 2 level of interaction for more information and analysis of most-fit or closed-fit.

Else

In the case of indeterminate disease

If (scope of automatic modification of the knowledge base is present)

incorporate with the doctor for the scope of determining exactly the disease along with its symptom list and through backward reasoning, match the actual symptom list with this. Incorporate some new medicine domain corresponding to this disease concept. Hence conclude the domain and go to L1.

Else

Regret in this trial after modifying knowledge base

Wait till the next trial and incorporation of new map between this disease and existing/new medicine.

Else if 
Add new symptom to the knowledge base;

If (scope of automatic modification of the knowledge base is present)

incorporate with a new map comprised this new found symptom and disease. This requires domain

Else specific knowledge. Hence a disease or list of diseases will be concluded.

Regret in this trial after modifying knowledge base

Wait till the next trial and incorporation of new map between this symptom and existing/new disease(s).

An illustrative control flow diagram is presented in the following figure.

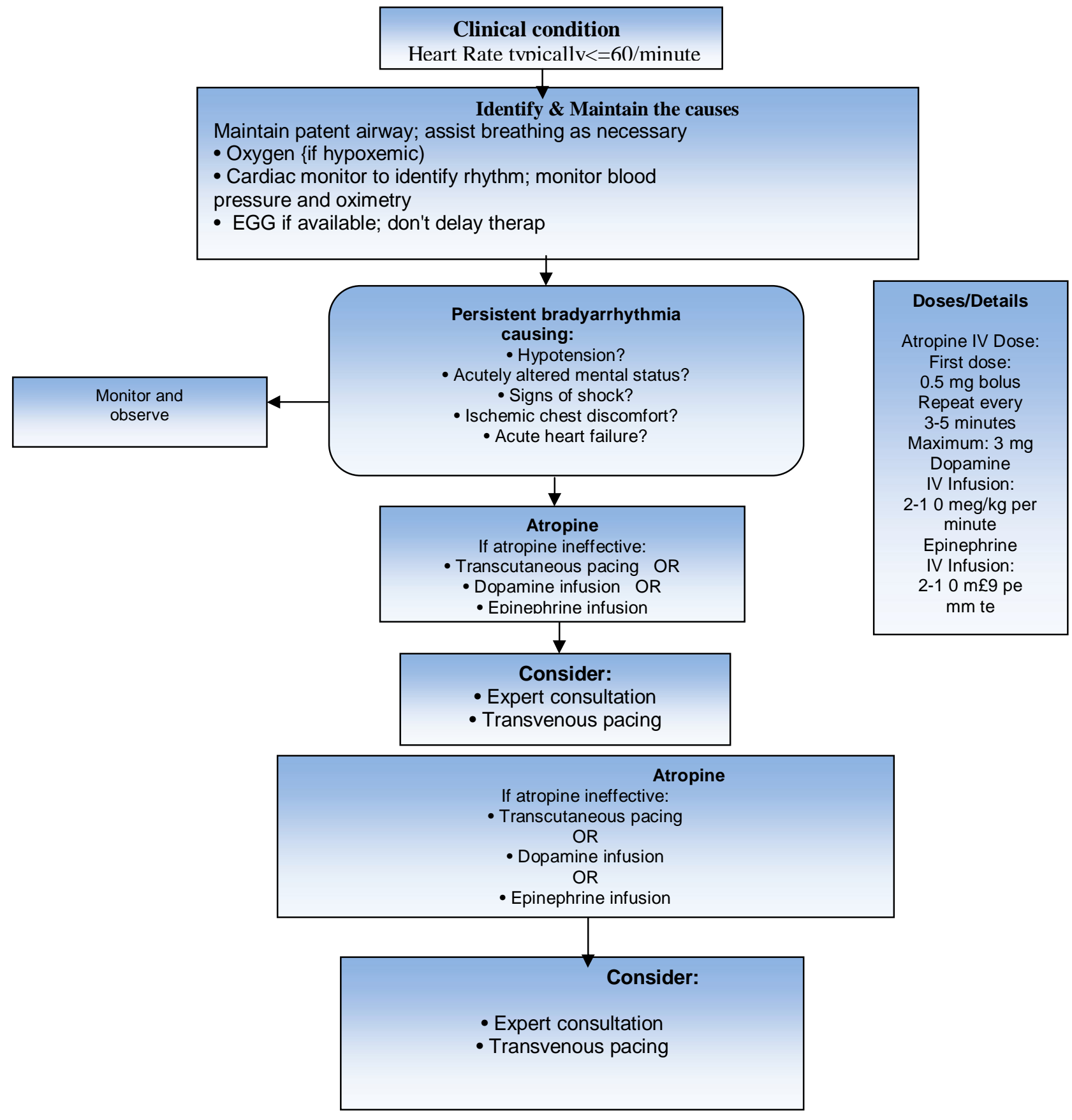

Fig.2 Normal Electrocardiogram and human heart. 


\section{RESUlt AND DisCUSSION}

The treatment convention is emphasized at the outset. Therapeutic Medicinary is the introduced treatment convention. The objective is not to research on how much effective and appropriate it is in the field of Medical Science.

The treatment convention is accepted and introduced only after analysis from technical viewpoint. The background detail of the treatment convention and the limitation / demerit of it (e.g. surgical cases can't be treated) are already discussed. Since, the domain of treatment has been specified as non-surgical (physical), so surgical treatment is only beyond the scope of the treatment.

But the questions, that why Therapeutic Medicinary is choosed as treatment convention and how much authentic is it from a technical viewpoint, deserves explanation. We have answered the first question at the beginning of the documentation where we have considered heart is the core of total physical system. Abnormal change in pulse rate, blood pressure can will be affected on total Physical disorder is nothing but a change or deviation from original state of vibration.

Sometimes specialized doctors are not available at any hospital or nursing home. Then RMO of the hospital or nursing home can manage the situation. i.e prescribe some general medicine to stable the situation till the specialized doctor came.

But lot of time patient can't reach nearby hospital or nursing home. because many of the reasons (no nearby hospital to rural area, sometimes communication problem, can afford the expenses of the private hospital or nursing home, but the major problem is doctors are most of the time not available at night, there times are wasted during waiting for the doctor \& travelling to hospital without the preliminary medicines, sometimes a minimal problem is turn up to major one shake of wastage of time, so sometimes when doctors came, the condition of the patients out of control of the doctor. )

Here, the device gets rid of wastage of time for determining basic basic medicines. it will prescribe the basic medicine according to his pressure formation. So the condition of the patient doesn't be major one till the doctor came or till the way to reach the doctor.

\section{A. Illustrations}

At first the device get the major four parameters of the program, these are Systolic, diastolic, pulse, age.

Now there will be a searching technique based on heuristic. There are seven conditions:

I. Pulse checking(heart rate)

II. Normal pressure

III. Low blood pressure

IV. Pre hypertension

V. High blood pressure (hypertension stage1)

VI. High blood pressure (hypertension stage2)

VII. Hypertensive crisis (immediate care needed)

VIII. Indeterminate

These are discussed in details:

\section{Pulse checking(heart rate):}

\section{i. Bradyarrythmia:}

When the heart rate is (heart rate $<=60 /$ minute) of any age. That means he/she is suffer from bradyarrythmia.i.e the heart is less than normal. it may be causes Hypotension, Acutely altered mental status, Signs of shock, Ischemic chest discomfort, acute heart failure.

In this condition the patient should be Maintain patent airway; assist breathing as necessary Oxygen \{if hypoxemic),Cardiac monitor to identify rhythm; monitor blood, pressure and oximetry, EGG if available; don't delay therapy.

Prescribed by device: "bradyarrythmia: Atropine IV Dose: First dose: $0.5 \mathrm{mg}$ Repeat every

3-5 minutes,Maximum: $3 \mathrm{mg}$. If Atropin ineffective Dopamine IV, Infusion:2-1 $0 \mathrm{meg} / \mathrm{kg}$ per minute, Epinephrine IV Infusion:-1 0 meg per minute, now go to expert consultation". 


\section{ii TACHYARRHYTHMIA:}

When the heart rate is (heart rate $>=120 /$ minute) of any age. That means he/she is suffer from tachyarrythmia.i.e the heart is more higher than normal. Also it may be causes Hypotension, Acutely altered mental status, Signs of shock, Ischemic chest discomfort, Acute heart failure..

Prescribed by device: "Tachyarrhythmia: Initial recommended doses: Narrow regular: 50-1 $00 \mathrm{~J}$

Narrow irregular:120-200 J biphasic or $200 \mathrm{~J}$ monophasic Wide regular: $100 \mathrm{~J}$ Wide irregular: defibrillation dose Adenosine IV Dose: First dose: $6 \mathrm{mg}$ rapid IV push; follow with NS flush. Second dose: $12 \mathrm{mg}$ if required.Antiarrhythmic Infusions for Stable Wide-QRS Tachycardia. over 5 minutes. now go to expert consultation".

\section{Normal pressure}

When the blood pressure lies between ((systolic $==120) \|($ diastolic $==80)$ ) of any age.and also his/her pulse is normal. Prescribed by device: "your pressure is in control and normal.no remedy required.STAY BLESSED".

\section{Low blood pressure}

When the blood pressure lies between $(($ systolic < 120)\|(diastolic < 80)) then the patient suffering from low blood pressure. So there need a medicine. But before prescribe any medicine there will be checking of age. because according to age the medicine will be prescribed with low dose or high dose. and the pulse of the patient is checked before . i.e is he/she suffering from bradyarrythmia or tachyarrythmia,then there will be separate medicine for that. which is prescribe earlier.

When the age lies between 30 to 49 .Then Prescribed by device: "low pressure: Mephentine $(30 \mathrm{mg}), 1 /$ day" .

When the age lies between 50 to 65 . Then Prescribed by device: " low pressure:Midodrine, 1/day"

When the age lies between 66 to 80 .Then Prescribed by device: " low pressure: Mephentine (15 mg,1/day).

\section{Pre hypertension:}

When the blood pressure lies between $(($ systolic $==120) \& \&($ systolic $==139) \|($ diastolic $==80) \& \&($ diastolic $==89))$ then the patient suffering from pre hypertension. So there need a medicine. But before prescribe any medicine there will be checking of age. because according to age the medicine will be prescribed with low dose or high dose. and the pulse of the patient is checked before . i.e is he/she suffering from bradyarrythmia or tachyarrythmia,then there will be separate medicine for that. which is prescribe earlier.

But in case of pre hypertension, am not suggesting any medicine,because many of frequent reasons(due to environment, stress, mental conditions, mood swing ) it may be occure. so there not needed any medicine, I think so.it may be overcome by regular life style.

So at any age, Prescribed by device:"pre high pressure: Cut back on dietary salt/sodium.Exercise regularly".

V. High blood pressure (hypertension stage1)

When the blood pressure lies between $(($ systolic $==140) \& \&($ systolic $==159) \|($ diastolic $==90) \& \&($ diastolic $==99))$ then the patient suffering from high blood pressure(hypertension 1). So there need a medicine. But before prescribe any medicine there will be checking of age. because according to age the medicine will be prescribed with low dose or high dose. and the pulse of the patient is checked before . i.e is he/she suffering from bradyarrythmia or tachyarrythmia,then there will be separate medicine for that. which is prescribe earlier.

When the age lies between 30 to 49 . Then Prescribed by device: " stage1 high pressure: Angiotensinconverting enzyme (ACE) inhibitors."

When the age lies between 50 to 80 .Then Prescribed by device: "stage1 high pressure:Diuretics (water pills). Angiotensin-converting enzyme (ACE) inhibitors."

\section{High blood pressure (hypertension stage2):}

When the blood pressure lies between ((systolic $>=160) \|($ diastolic $>=100))$ then the patient suffering from high blood pressure(hypertension 2). So there need a medicine. But before prescribe any medicine there will be 
checking of age. because according to age the medicine will be prescribed with low dose or high dose. and the pulse of the patient is checked before . i.e is he/she suffering from bradyarrythmia or tachyarrythmia,then there will be separate medicine for that. which is prescribe earlier.

If the age lies between 30 to 49, then Prescription by device is "stage2 high pressure: Angiotensin II receptor blockers."

If the age lies between 50 to 80 ., then Prescription by device is "stage2 high pressure:Diuretics (water pills).Angiotensin II receptor blockers."

\section{Hypertensive crisis (immediate care needed)}

When the blood pressure lies between ((systolic >=180)\|(diastolic >=110)) then the patient suffering from hypertensive crisis(immediate care needed). So there need a medicine. But before prescribe any medicine there will be checking of age. because according to age the medicine will be prescribed with low dose or high dose. and the pulse of the patient is checked before . i.e is he/she suffering from bradyarrythmia or tachyarrythmia,then there will be separate medicine for that. which is prescribe earlier.

If the age lies between 30 to 49, then Prescription by device is" crisis high pressure: Diuretics (water pills)+Beta blockers."

If the age lies between 50 to 80 , the Prescription by device is:"crisis high pressure: Diuretics (waterpills)+Angiotensin II receptor blockers.Beta blockers. "

\section{Indeterminate cases:}

When some symptoms are beyond its knowledge base then it will prescribe a basic medicine "Diuretics (water pills)" to remain constant the equilibrium of heart system.And also alert the patient "The problem or disease is indeterminant, Medicine can not be determine?recognized,!! contact physically to nearest doctor as soon as possible."

It will be practically implemented by keeping some weights to each symptom to determine precedence as number of diseases can have same symptoms with different intensity.

\section{B. Details of healing: approved medicine}

1. Atropine IV Indications and Usage for Atropine:

Atropine Sulfate Injection, USP, is indicated for temporary blockade of severe or life threatening muscarinic effects, e.g., as an antisialagogue, an antivagal agent, an antidote for organophosphorus or muscarinic mushroom poisoning, and to treat bradyasystolic cardiac arrest.

Adult Dosage:

Table 1: Recommended Dosage

\begin{tabular}{l|l|l}
\hline Use & $\begin{array}{l}\text { Dose } \\
\text { (adults) }\end{array}$ & Repeat \\
\hline Antisialagogue or other antivagal & 0.5 to $1 \mathrm{mg}$ & $1-2$ hours \\
\hline $\begin{array}{l}\text { Organophosphorus or muscarinic mushroom } \\
\text { poisoning }\end{array}$ & 2 to $3 \mathrm{mg}$ & $20-30$ minutes \\
\hline Bradyasystolic cardiac arrest & $1 \mathrm{mg}$ & $\begin{array}{l}3-5 \text { minutes; } 3 \mathrm{mg} \\
\text { maximum total dose }\end{array}$ \\
\hline
\end{tabular}

Pediatric Dosage: Dosing in pediatric populations has not been well studied. Usual initial dose is 0.01 to 0.03 $\mathrm{mg} / \mathrm{kg}$.Dosage Forms and Strengths:Injection: $0.05 \mathrm{mg} / \mathrm{mL}$ and $0.1 \mathrm{mg} / \mathrm{mL}$ in Ansyr ${ }^{\mathrm{TM}}$ Plastic Syringes.

2. Dopamine IV:

Dopamine is a medication form of a substance that occurs naturally in the body. It works by improving the pumping strength of the heart and improves blood flow to the kidneys. 
Usual Adult Dose of Dopamine for Shock: Initial dose: 1 to $5 \mathrm{mcg} / \mathrm{kg} / \mathrm{min}$ by continuous IV infusion. Titrate to desired response. Administration at rates greater than $50 \mathrm{mcg}$ per $\mathrm{kg}$ per minute have been used safely in serious situations.

Usual Pediatric Dose for Nonobstructive Oliguria: less than 1 month: 1 to $20 \mathrm{mcg} / \mathrm{kg} / \mathrm{min}$ by continuous IV infusion, titrated to desired response.

Usual Pediatric Dose for Shock:

less than 1 month: 1 to $20 \mathrm{mcg} / \mathrm{kg} / \mathrm{min}$ by continuous IV infusion, titrated to desired response. 1 month or older: 1 to $20 \mathrm{mcg} / \mathrm{kg} / \mathrm{min}$ by continuous IV infusion, titrated to desired response. Maximum of 50 $\mathrm{mcg} / \mathrm{kg} / \mathrm{min}$.

\section{Epinephrine IV:}

Epinephrine should be given at the first sign of anaphylaxis regardless of cause. The decision as to whether or not to administer epinephrine via intramuscular or intravenous administration depends upon the severity of the episode, and of course, whether or not the patient is in shock, which may make intravenous administration necessary.

However, in most instances, epinephrine can be administered intramuscularly. The dose in an adult is 0.3 to $0.5 \mathrm{cc}$. The standard intramuscular dose is a 1:1,000 concentration. This should be given in the lateral aspect of the thigh by intramuscular injection. The dose can be repeated every 5 to 15 minutes, depending upon the response, for three to four doses. The same is true for children except the dose is $0.01 \mathrm{mg} / \mathrm{per} / \mathrm{kg}$.

\section{4. $\quad$ Adenosine IV:}

Intravenous Aden card (adenosine injection) is indicated for the following.

It is important to be sure the Adenocard solution actually reaches the systemic circulation. Adult Patients:

Initial dose: $6 \mathrm{mg}$ given as a rapid intravenous bolus (administered over a 1-2 second period).

Repeat administration: If the first dose does not result in elimination of the supraventricular tachycardia within 1-2 minutes, $12 \mathrm{mg}$ should be given as a rapid intravenous bolus. This $12 \mathrm{mg}$ dose may be repeated a second time if required.

\section{Pediatric Patients:}

Pediatric Patients with a Body Weight < $50 \mathrm{~kg}$ :

Initial dose: Give 0.05 to $0.1 \mathrm{mg} / \mathrm{kg}$ as a rapid IV bolus given either centrally or peripherally. A saline flush should follow.

Pediatric Patients with a Body Weight $\geq 50 \mathrm{~kg}$ : Administer the adult dose.

Doses greater than $12 \mathrm{mg}$ are not recommended for adult and pediatric patients.

5. $\quad$ Procainamide IV:

Procainamide injection is used to treat irregular heartbeats and to slow an overactive heart. When the heart has a normal heartbeat (rhythm), it will work more efficiently. Procainamide works by slowing the nerve impulses in the heart and reducing the sensitivity of heart tissues.

Applies to the following strength(s): $250 \mathrm{mg} ; 375 \mathrm{mg} ; 500 \mathrm{mg} ; 100 \mathrm{mg} / \mathrm{mL} ; 500 \mathrm{mg} / \mathrm{mL} ; 750 \mathrm{mg} ; 1000 \mathrm{mg} ; 500$ $\mathrm{mg} / 12$ hours ; $1000 \mathrm{mg} / 12$ hours.

6. Amiodarone IV:

Cordarone I.V. is indicated for initiation of treatment and prophylaxis of frequently recurring ventricular fibrillation and hemodynamically unstable ventricular tachycardia in patients refractory to other therapy.also can be used to treat patients with VT/VF for whom oral Cordarone is indicated, but who are unable to take oral medication.

7. $\quad$ Mephentine $(30 \mathrm{mg} / 15 \mathrm{mg})$ :

Mephentine (30 mg) Mephentine $(30 \mathrm{mg}$ ) is a cardiac stimulant, prescribed for hypotension (low blood pressure).

Indications: Mephentine (30 mg) is a cardiac stimulant, prescribed for hypotension (low blood pressure).

Contraindications: Contraindicated in patients with low blood pressure induced by chlorpromazine, concurrent use with any MAOIs ( Monamine Oxidase Inhibitors).

Active ingredients: Mephentermine .

\section{Midodrine:}


Midodrine ProAmatine ${ }^{\circledR}$ (Midodrine hydrochloride) Tablets forms an active metabolite, desglyMidodrine, that is an alpha1-agonist, and exerts its actions via activation of the alpha-adrenergic receptors of the arteriolar and venous vasculature, producing an increase in vascular tone and elevation of blood pressure.

Active ingredients: midodrine .

9. Angiotensin-converting enzyme (ACE) inhibitors:

Angiotensin-converting enzyme (ACE) inhibitors help relax blood vessels.it prevents an enzyme in your body from producing angiotensin II, a substance in your body that affects your cardiovascular system by narrowing your blood vessels and releasing hormones that can raise your blood pressure.

10. Diuretics (water pills):

For high blood pressure, diuretics, commonly known as "water pills," help your body get rid of unneeded water and salt through the urine. Getting rid of excess salt and fluid helps lower blood pressure and can make it easier for your heart to pump. Diuretics may be used to treat a number of heart-related conditions, including high blood pressure, heart failure, kidney and liver problems, and glaucoma.

11. Angiotensin II receptor blockers:

Angiotensin II is a natural substance in your body that affects your cardiovascular system in many ways, such as by narrowing your blood vessels. This narrowing can increase your blood pressure and force your heart to work harder \& also starts the release of a hormone that increases the amount of sodium and water in your body, which can lead to increased blood pressure.

12. Beta blockers:

Beta blockers, also called beta-adrenergic blocking agents, treat a variety of conditions, such as high blood pressure, glaucoma and migraines. Find out more about this class of medication.

Beta blockers work by blocking the effects of the hormone epinephrine, also known as adrenaline. When you take beta blockers, the heart beats more slowly and with less force, thereby reducing blood pressure. Beta blockers also help blood vessels open up to improve blood flow.

The proposed remedies the above medicines which are medically approved, recommended by medical practitioner.

\section{IV.CONCLUSION}

The conclusive remarks may be tangibly presented as follows:

- The advantages of the diagnosis are used to detect any worse condition of physical status of heart.

- The follow-up technique is used to precaution the problem by giving a alert message \& suggesting immediate medicine which is clinically approved.

- Useful for non-specialist person/patient him/her-self in the analysis of probable heart failure.

- The detection method done by the sensor, which may be in wrist-watch format this simulation results will be then proved that the developed algorithm has been shown to be a powerful technique device in pre-detection of heart diseases.

- There will be different types of sensor, blood pressure sensor, and pulse sensor.

- Mobile can be identified as pervasive /ubiquitous device to alert in this work.

\section{REFERENCES}

[1] S. Gupta, A. Datta, "Cognitive Systems incorporating Artificial Intelligence: A behavioral model of Music Therapy Treatment Convention", Proceedings of the $7^{\text {th }}$ International Conference on Cognitive Systems (ICCS) 2005, ISBN: 81-903047-2-0, 52, pp. 1-14, 2005.

[2] J Mackay, G Mensah "Prevention of Cardiovascular Disease Guidelines for assessment and management of Cardiovascular risk", World Health Organization 2007."Atlas of Heart Disease and Stroke”, 2004 World Health Organization, Geneva.

[3] Cardiac physiology from Wikipedia, the free encyclopedia.

[4] Taolue Chen, Marco Diciolla, Marta Kwiatkowska, Alexandru Mereacre "A Simulink Hybrid Heart Model for Quantitative Verification of Cardiac Pacemakers", Department of Computer Science University of Oxford, UK .

[5] Gabriela Ortiz-León, Marta Vílchez-Monge, and Juan J. Montero-Rodríguez "Simulations of the Cardiovascular System Using the Cardiovascular Simulation Toolbox", , Costa Ricamvilchez@itcr.ac.cr 3. Escuela de Ingeniería Electrónica, Instituto Tecnológico de Costa RicaCartago, Costa Ricajjmontero@itcr.ac.cr 
[6] Arduino function (//www.arduino.cc/en/Reference/Serial)

[7] Prakash D, Uma Mageshwari T, Prabakaran K, and Suguna, "Detection of Heart Diseases by Mathematical lnteligence Algorithm Using Phonocardiogram Signals" , Anna University Regional Centre Coimbatore,Coimbatore 641047, Tamilnadu, India.

[8] iHealth ${ }^{\mathrm{TM}}$ Wireless Blood Pressure Monitor (BP5)

[9] real-time systems design and analysis tools for the practitioner fourth edition, phillip a. laplante, seppo j. ovaska, ieee press.

[10] Blood Pressure Monitor Fundamentals and Design by: Santiago Lopez

[11] Medicine books based on medical knowledge.a Ventricular Arrhythmias In Apparently Normal Hearts, An Issue Of Cardiac Electrophysiology Clinics,Frank Bogun b.Advanced Heart Failure, An Issue of Heart Failure Clinics, 1st Edition,Authors: James Fang \& Michael Givertzc.Pulmonary Hypertension, An Issue of Cardiology Clinics, 1st Edition,Author: Ronald J. Oudiz

[12] Dong-Hyun Nam, You-Sik Hong, Woo-Beom Lee, Il-Ho Son, Keun-Ho Kim and Jong-Gu Ch," Measurement of Blood Pressure Using an Arterial Pulsimeter Equipped with a Hall Device Sang-Suk Lee",E-Mails: yshong@sangji.ac.kr (Y.-S.H.); beomlee@sangji.ac.kr (W.-B.L.)4 Department of Western-Eastern Biomedical Engineering, Sangji University, Wonju 220-702, Korea; E-Mail: choijong9@ sangji.ac.kr 